\title{
IN THE AUGUST 2008 ISSUE OF CLINICS
}

\author{
Mauricio Rocha-e-Silva, Editor
}

doi: $10.1590 / \mathrm{S} 1807-59322008000400001$

In this issue of CLINICS we would like to highlight a study by Karadeniz et al. on accumulation of oxidized low-density-lipoprotein in a model of murine liver fibrosis induced by cholestasis. Following a 21 day bile duct ligation, authors report higher levels of malondialdehyde, lower levels of superoxide-dismutase, and positive oxLDL cholesterol staining were found in liver homogenates of jaundiced rats, as compared to sham operated animals. They suggest that oxLDLs are produced as an intermediate agent during exacerbated oxidative stress or, alternatively, that they contribute to mechanisms underlying the process of liver fibrosis.

This issue includes five reports on original research related to the cardiovascular system:

da Luz et al. studied 374 high risk patients submitted to coronary angiography and found that a high ratio of triglycerides and HDL-cholesterol is a powerful and independent marker of coronary disease: the increment of one quartile of that ratio is followed by a $30 \%$ increase in the frequency of extensive CAD, adjusted for other lipid variables and is the greatest influence among lipid variables.

Bocalini et al. analyzed the effect of physical exercise on functional and quality of life in 22 patients with grade II and III heart failure (NYHA). Twenty two patients were randomized into training vs. non-training groups and the former went through a 6-months exercise program. Fitness and quality of life indices were evaluated and showed that guided and monitored physical exercise is safe and able to improve the functional capacity and quality of life in heart failure patients.

Carvalho et al. studied 25 patients in optimized beta-blocked heart failure with an average left ventricular ejection fraction of $30 \%$, and fourteen controls to evaluate heart rate dynamics during a treadmill cardiopulmonary exercise test. They found that no patient in the heart failure group reached the maximum heart rate for their age, despite the fact that the percentage increase of heart rate was similar to sedentary normal subjects. They proposed that a heart rate increase in optimized beta-blocked heart failure patients during cardiopulmonary exercise test over $65 \%$ of the maximum age-adjusted value should be considered an effort near the maximum.

Costa e Silva et al. compared conventional and transdisciplinary care in a tertiary outpatient clinic for patients after their first acute myocardial infarction. A total of 153 patients were allotted to conventional $(n=75)$ or transdisciplianry $(n=78)$ care. Patients were followed for 180 days. The primary outcome was clinical improvement, as evaluated by an index including reduction of body weight,

Hospital das Clínicas, Faculdade de Medicina da Universidade de São Paulo - São Paulo/SP, Brazil.

mrsilva36@hcnet.usp.br lowering of blood pressure, smoking cessation, increase in physical activity, and compliance with medication. Compliance with diet and visits was higher for transdisciplinary care vs. conventional care; however, the transdisciplinary approach did not provide more clinical benefits than the conventional approach.

Casella et al. describe the testing of a practical protocol to measure common carotid intima-media thickness that uses the combined values of two longitudinal examination angles to increase sensitivity. Duplex scan examination of carotid vessels was performed and the intima-media thickness of 407 common carotids were measured in three angles: transversal, longitudinal posterolateral, and anterolateral. In addition to numbers obtained from the three angles of measurement, a fourth visual perspective was obtained by combining the intima-media thickness results of longitudinal posterolateral and anterolateral longitudinal views, and considering the thickest wall measurement. Authors claim that the protocol is a practical method for obtaining common carotid artery intima-media thickness measurements. The combined longitudinal posterolateral and anterolateral longitudinal views provide a more sensitive evaluation of the inner layers of the carotid walls than isolated longitudinal views.

Five studies cover problems in anatomy and function of the musculo-skeletal system:

Lucareli and Greve describe knee joint dysfunctions that influence gait in cerebrovascular injury in a study including 66 adult men and women with a diagnosis of either right or left hemiparesis, resulting from ischemic cerebrovascular injury; all underwent threedimensional gait evaluation and an the angular kinematics of the joint knee were selected for analysis. Relevant clinical characteristics included mechanisms of loading response in the stance, knee hyperextension in single stance, and reduction of the peak flexion and movement amplitude of the knee in the swing phase. Authos suggest that these mechanisms should be taken into account when choosing the best treatment.

Milani et al. checked on the correlation between the degree of gynoid lipodystrophy (cellulite) and lumbar hyperlordosis in 50 volunteers evaluated through digital photography, palpation, and thermograph, but no correlation was found between the angle of lumbar lordosis and degree of cellulite.

Rosito et al. studied 49 patients submitted to an acetabular component revision of a total hip arthroplasty, using impacted human $(n=26)$ and bovine freeze-dried $(n=25)$ cancellous bone grafts, and a reinforcement device. No clinical/radiographic differences were found between the groups and both showed an overall rate of $88.5 \%$ and $76 \%$ of graft incorporation. 
Rai et al. studied anatomical variability of the omohyoid muscle and its clinical relevance in 35 cadavers. A double omohyoid was present in one cadaver; the inferior belly originated from the clavicle in three cadavers; the superior belly merged with the sternohyoid in two cadavers, and the omohyoid received additional slips from the sternum in one cadaver. Standard attachment and position of the omohyoid was observed in the remaining cadavers. Such variations are important because of a close relation of the muscle to the large vessels and brachial plexus. Because of the direct adhesion of the intermediate tendon to the anterior wall of the internal jugular vein and its connection with it through a thin lamina of the pretracheal layer of the cervical fascia, the contraction of the omohyoid muscle has a direct effect on the lumen of this vessel.

Aragão et al. performed metric measurements to verify the attachment levels of the medial patellofemoral ligament in 17 knees from 10 human cadavers, which were intact and showed no macroscopic signs of injuries. The medial patellofemoral ligament was present in $88 \%$ of the knees; the width of its insertion ranged from 16 to $38.8 \mathrm{~mm}$, with a mean of $27.90 \mathrm{~mm}$, and its mean length was $55.67 \mathrm{~mm}$. The margins of the ligament were concave or rectilinear. At the upper margin, the concave form predominated and was better characterized, while at the lower margin, the rectilinear form predominated.

Three papers deal with gynecologic and fertility issues:

Castro et al. report on a single-blind, randomized, controlled trial of pelvic floor muscle training, electrical stimulation, vaginal cones, and no active treatment in the management of stress urinary incontinence in which the effectiveness of these 3 procedures was compared. It was found that pelvic floor exercises, electrical stimulation, and vaginal cones are equally effective treatments and far superior to no treatment in women with urodynamic stress urinary incontinence. Pelvic floor training is recommended as the premier strategy in this situation.

Lobo et al. evaluated the effects of estrogen treatment in combination with gestrinone on an experimental rat model of endometriosis. Uterine transplants were attached to the peritoneum of female Wistar rats via a surgical autotransplantation technique. A high dose of estrogen caused macroscopic increases in the endometrial implant group compared with other groups, which were similar to increases in the proestrus phase. A low dose showed morphometric development of implants, such as an increase in number of endometrial glands, leukocyte infiltration and mitosis. Gestrinone antagonized both doses of estrogen. Our findings suggest that gestrinone antagonizes estrogen's effects on rat peritoneal endometrial implants

Ibrahim et al. studied the antioxidant effect of alpha lipoic acids on the sperm quality of semen from six Boer bucks. Seminal analysis of the baseline before and after incubation of samples with a series of doses of Alpha lipoic acids was performed. Sperm motility rate was improved after incubation with Alpha lipoic acids at a concentration of $0.02 \mathrm{mmol} / \mathrm{ml}$. This concentration was also capable of reducing DNA damage.

Two studies address problems in Ophthalmology:

Giampani Jr et al. report on the long term efficacy and safety of trabeculectomy with mitomycin c for childhood glaucoma: a study of results with long-term follow-up of 114 patients. They conclude that trabeculectomy with mitomycin is safe and effective for short-term or long-term treatment of congenital or developmental glaucoma. The frequency of bleb-related endophthalmitis was no higher in these patients than that described in adults.
Moraes and Susanna Jr. report on the correlation between the water drinking test and modified diurnal tension curve in untreated glaucomatous eyes, and conclude that intraocular pressure peaks detected during the Water Drinking Test could be used in clinical practice to estimate the peaks observed during the Modified Diurnal Tension Curve and to assess the status of the eye's outflow facility.

We also publish 2 orignal reports on sepsis:

Rezende et al. describe the epidemiology of severe sepsis in the emergency department and difficulties in the initial assistance for 342 out of 5332 patients admitted to the Emergency Department of a public Hospital. Authors find that the occurrence rate of severe sepsis in the emergency department was $6.4 \%$, and the rate of sepsis diagnosed by the emergency department team, as well as the number of patients transferred to the ICU was very low. Educational campaigns are important to improve diagnosis and, hence, treatment of severe sepsis.

Freitas et al. analyzed the impact of duration of organ dysfunction on the outcome of 56 patients with severe sepsis and septic shock; the duration of organ dysfunction prior to diagnosis was correlated with mortality. In a multivariate analysis, only duration of organ dysfunction persisting longer than 48 hours correlated with mortality. These findings suggest that the diagnosis of organ dysfunction is not being made in a timely manner. The time elapsed between the onset of organ dysfunction and initiation of therapeutic intervention can be quite long, and this represents an important determinant of survival in cases of severe sepsis and septic shock.

Two studies in infectology cover problems relating to Leishmaniois and HIV/AIDS:

Medeiros et al. studied the involvement of Leishmania (L.) amazonensis in American tegumentary leishmaniasis in the state of São Paulo, Brazil. DNA sequencing permitted the identification of a particular 15bp fragment (5'...GTC TTT GGG GCA AGT... 3') in all samples. Analysis by the neighbor-joining method showed the occurrence of two distinct groups related to the genus Viannia $(V)$ and Leishmania $(L)$. These results confirm the pattern of distribution and possible mutations of these species, as well as the change in the clinical form presentation of ATL in the state of São Paulo.

Soeiro et al. performed a post-mortem histological pulmonary analysis of the pathology observed in 250 autopsies of HIV/AIDS patients. This report covers a wide range of findings and is the first autopsy study to include demographic data, etiologic diagnosis, and respective histopathological findings in patients with HIV/ AIDS and acute respiratory failure. Further studies are necessary to elucidate the complete pulmonary physiopathological mechanism involved with each HIV/AIDS-associated disease.

One study covers the problem of posttraumatic stress disorder:

Assis et al. evaluated physical activity habits in 50 patients with posttraumatic stress disorder and found substantial changes in habits following the onset of PTSD. While more than half of the patients participated in physical activities prior to Posttraumatic Stress Disorder onset, there was a significant reduction in their participation afterwards. The justifications for stopping physical activities or sport participation were lack of time and lack of motivation. The findings demonstrate that patients with Posttraumatic Stress Disorder have low levels of participation in sports or physical activities. 